\title{
Improving surgical outcomes in advanced ovarian cancer: a UK centre quality improvement strategy
}

James May ( $\nabla$ james.may@nhslothian.scot.nhs.uk)

NHS Lothian https://orcid.org/0000-0002-3348-7199

Mario Congiu

NHS lothian

\section{Scott Fegan}

NHS lothian

Nidal Ghaoui

NHS Lothian

David Anderson

NHS Lothian

Mhairi Collie

NHS Lothian https://orcid.org/0000-0001-5583-0659

Tzyvia Rye

University of Edinburgh

Charlie Gourley

University of Edinburgh https://orcid.org/0000-0002-2377-7221

Cameron W Martin

NHS Lothian

Research article

Keywords: Ovarian Cancer; Cytoreductive surgery; Oncology; Residual disease

Posted Date: July 3rd, 2019

DOI: https://doi.org/10.21203/rs.2.10917/v1

License: (c) (i) This work is licensed under a Creative Commons Attribution 4.0 International License.

Read Full License 


\section{Abstract}

Purpose National outcomes for epithelial ovarian cancer in the UK are inferior to those in other European countries. This study aimed to assess the impact of a multidisciplinary approach to cytoreductive surgery on residual disease status and progression free survival in a single UK region. Methods Consecutive patients undergoing surgery for stage III/IV epithelial ovarian cancer between November 2011 and November 2015 in South East Scotland were analysed. Regular multidisciplinary surgery was established on 1st November 2013. Outcomes for patients diagnosed in the two years prior to (group 1) and following this change (group 2) in practice were examined. Results 224 consecutive patients underwent surgery. There was no difference between groups in terms of age, FIGO stage or histological subtype. Patients in group 2 were more likely to undergo primary surgery $(p=0.01)$ and bowel resection $(p=0.002)$. Rates of optimal cytoreduction were not significantly different between group 1(69.6\%) and group 2 (66.1\%) $(p=0.67)$. The upper abdomen was the most common site of residual disease. No difference in PFS (median PFS; 15.5 vs. 14.0 months, $p=0.13)$, morbidity $(p=0.82)$ or mortality $(p=1.00)$ was identified. PFS was improved in those patients undergoing primary surgery compared to neoadjuvant chemotherapy $(p=0.04)$. Conclusion Robust quality improvement strategies are required to overcome historic barriers to cytoreductive surgery in the UK. Comprehensive attempts at debulking in the upper abdomen are required if rates of complete cytoreduction are to be improved. We have extended the multidisciplinary model in our institution to include upper abdominal surgery and anticipate an improvement in cytoreduction.

\section{Background}

Ovarian cancer is the $6^{\text {th }}$ most common female cancer and the most lethal gynaecological malignancy in the UK, responsible for 4128 deaths in 2014[1]. Surgical effort is consistently identified as the most important modifiable determinant of prognosis in epithelial ovarian cancer (EOC), with resection to zero macroscopic residual disease associated with a significant survival benefit $[2,3]$. There are minimal published data regarding surgical practice in the management of advanced EOC in the UK where five-year survival is inferior to survival in other European countries [4]. The validity of survival information as a measure of health care performance is debated since large variations in coverage of cancer registry information exist internationally, with virtually complete coverage in the UK, yet survival in the UK remains inferior even when compared only to countries where high quality, comprehensive cancer registry data exist [5]. It has been suggested that, if UK survival was equal to survival rates in other European countries, an additional 478 lives per year could be saved[6]. A 2013 survey of gynaecological oncologists in the UK demonstrated significant variation in practice [7]. The survey identified structural resources and surgical skills as barriers to the development of more radical surgery. The authors concluded that, although poor UK outcomes are multifactorial, surgical performance is likely to directly contribute to reduced survival in EOC. A further survey of UK practice suggested that in many centres, complete cytoreduction was not the surgical goal [8]. In an effort to address barriers of infrastructure and surgical training, a quality improvement strategy consisting of a multidisciplinary (gynaecology oncology/colorectal/urology) surgical approach to EOC was established in our centre in 2013 with the 
aim of optimising surgical skills to improve rates of maximal surgical cytoreduction and minimising rates of postoperative morbidity. The aim of this study was to assess the impact of this new service model on residual disease rates and outcomes as well as surgical morbidity and mortality.

\section{Methods}

The South East of Scotland Cancer Network (SCAN) serves a population of 1.4 million across four NHS health boards. All women undergoing primary or interval debulking surgery for advanced EOC (FIGO stage III/IV) within SCAN, from $1^{\text {st }}$ November 2011 to $31^{\text {st }}$ October 2015 were included in the analysis. Secondary surgery for recurrent EOC was not included. In all cases histological diagnosis was confirmed on central pathological review by a gynaecologic pathologist. Operations were performed via a midline laparotomy by a gynaecological oncologist. Regular multidisciplinary surgery inclusive of urological and colorectal surgeons was established on $1^{\text {st }}$ November 2013. Outcomes for patients diagnosed in the two years prior to and following this change in practice were examined (Group 1: $1^{\text {st }}$ November $2011-31^{\text {st }}$ October 2013, Group 2: $1^{\text {st }}$ November $2013-31^{\text {st }}$ October 2015). Primary outcomes were residual disease status following surgery and progression free survival (PFS). Secondary outcomes were surgical morbidity and mortality.

Patient management was determined by discussion at the gynaecology oncology multidisciplinary team meeting (MDM) consisting of consultants in gynaecological oncology surgery, medical and clinical oncology, gynaecological pathology, gynaecological radiology and clinical nurse specialists. Primary surgery was considered the standard treatment. Patients were treated with neoadjuvant chemotherapy if performance status was poor and/or disease was considered to be unresectable based on cross sectional imaging. Patients proceeded to interval debulking surgery if disease was considered stable or demonstrated evidence of response to 3-4 cycles of neoadjuvant chemotherapy (NACT) (carboplatin +/paclitaxel). Patients were discussed at a secondary MDM prior to proceeding with surgery consisting of gynaecology oncology, colorectal and urology surgeons, medical oncologists and gynaecological radiologist.

Data were recorded prospectively on the University of Edinburgh ovarian cancer database. Variables recorded included age at diagnosis, stage (International Federation of Gynecology and Obstetrics 2014 classification), histological subtype, treatment strategy (primary surgery versus neoadjuvant chemotherapy) and residual disease status. Site of residual disease, morbidity and mortality data were collated from review of patient health records.

Data collection, storage and analysis were in accordance with local governance protocols. As a service evaluation this study is exempt from the requirement for research ethical review and was authorised by the local Caldicott guardian. Survival data was calculated using the Kaplan-Meier Method and differences in PFS were analysed by the log-rank test. Categorical variables were compared using Fisher's exact test. Continuous variables were compared using the Student's $t$ test. All statistical analysis was performed using GraphPad Prism Software (Version 7 for Windows, La Jolla, California, USA). 


\section{Results}

A total of 224 consecutive patients who had surgery for advanced EOC were identified during the study period. Median age was 66 years (range $19-89$ ) in both groups. The majority of women were diagnosed with high grade serous carcinoma (79.0\%). Most patients had stage III disease $(71.9 \%)$. Patient characteristics are summarized in Table 1. Median follow up for the entire cohort was 23 months (range 1-60). Median follow up was longer in group 1, 37 months, compared to group 2, 18 months. At the time of analysis 90 (39.3\%) women were known to have died: 57 women from group $1(50.1 \%)$ and 34 (29.1\%) from group 2.

Across the entire study population, we achieved cytoreduction to no macroscopic disease in $43.8 \%$ cases. Resection to less than $1 \mathrm{~cm}$ residual disease was achieved in a further $24.1 \%$ of cases. Following the implementation of regular multidisciplinary operating lists, the number of patients undergoing primary surgery increased significantly from $39.3 \%$ in group 1 to $56.3 \%$ in group $2(p=0.01)$. In group 1 , zero macroscopic residual disease was achieved in $47.3 \%$ of patients and resection to $<1 \mathrm{~cm}$ residual disease in a further $22.3 \%$. In group 2, zero macroscopic residual disease was achieved in $40.2 \%$ and resection to $<1 \mathrm{~cm}$ in a further $25.9 \%$. No statistical difference was achieved in either complete $(p=0.35)$ or optimal $(<1 \mathrm{~cm})$ resection rates $(p=0.64)$ between the two groups. Residual disease status is summarized in table 2. In $12 / 224$ (5.4\%) cases, patients underwent open/close laparotomy due to unresectable disease $(7.1 \%$ in group 1 versus $3.6 \%$ in group 2). Excluding this group, 114 patients had residual disease. The upper abdomen was the most common site of residual disease in both groups and was the only site of residual disease in $18.0 \%(9 / 50)$ and $25 \%(16 / 64)$ in groups 1 and 2 , respectively $(\mathrm{p}=0.37)$. The proportion of women with residual disease in the pelvis fell from $13.7 \%$ in group 1 to $4.7 \%$ in group $2(p=0.11)$. Overall $31.3 \%(70 / 224)$ of patients underwent bowel resection, of which $26.8 \%(60 / 224)$ required large bowel resection +/- anastomosis. The rate of bowel resection increased from $21.4 \%(24 / 112)$ in group 1 to $41.1 \%(46 / 112)$ in group $2(p=0.002)$. Surgical procedures performed in addition to the standard procedures of hysterectomy, bilateral salpingo-oophorectomy and omentectomy are detailed in table 3 .

Thirty-six patients (15.7\%) were classified as FIGO stage IIIA/B on final staging. In the remaining 193 patients with FIGO stage IIIc/IV disease, there was no difference in rates of complete cytoreduction ( $44.8 \%$ in group 1 versus $35.1 \%$ in group $2, p=0.19)$ or resection to $<1 \mathrm{~cm}$ residual disease $(21.9 \%$ in group 1 versus $29.9 \%$ in group $2, p=0.25$ ) between the 2 groups.

Three patients died within 60 days of surgery, one from group 1 and 2 from group 2 . The 30-day surgical mortality rate was $0.4 \%(1 / 224) ; 60$-day mortality was $1.3 \%(3 / 224)$. One patient died on the $7^{\text {th }}$ postoperative day due to peritonitis secondary to ischaemic bowel, another died from disease progression on day 31 following an open/close laparotomy due to unresectable disease. The final patient died on day 45 due to rapid disease progression following complete macroscopic resection of disease. Twenty -three patients suffered major postoperative morbidity (10.3\%). Two patients required a return to theatre, one for postoperative haemorrhage, the second for small bowel obstruction secondary 
to internal hernia. There was no difference in morbidity rates between the two groups $(p=1.0)$. Morbidity is detailed in Table 4.

Median PFS for the entire cohort was 14.3 months. The median overall survival (OS) was not reached. There was no significant difference identified in progression free survival between the two groups. Median PFS in group 1 was 15.5 months and in group $2,13.7$ months $(p=0.13)$, although with less followup in group 2 this figure could subsequently change. Figure 1. Median PFS for patients undergoing primary surgery was 15.1 months compared to 13.8 months in patients undergoing neoadjuvant chemotherapy followed by delayed surgery. PFS was improved in patients undergoing primary surgery compared to delayed surgery when examined across the whole study population $(p=0.04)$ and in group 1 $(p=0.05)$ however no significant difference was identified in group $2(p=0.23)$. Figures $2-4$.

\section{Discussion}

Surgical cytoreduction is the most significant modifiable prognostic factor in EOC, with complete cytoreduction consistently associated with improved progression free and overall survival[9]. There is little published data regarding cytoreductive surgery in the UK and, this case series, represents only the third UK report of outcomes from cytoreductive surgery[10,11]. Surveys of UK practice in this regard have suggested significant variation in surgical practice with some centres reporting never achieving complete cytoreduction in either the primary $(12 \%)$ or interval surgical setting $(21 \%)[7,8]$. The issue of variation in surgical practice is not unique to the UK [12]. Factors contributing to ovarian cancer outcomes in the UK are undoubtedly complex and multifactorial, however an apparently less radical surgical approach in the UK may contribute to lower survival when compared to countries where more radical surgery has been widely adopted [4]. Structural resources and surgical training or skills have been highlighted as barriers to improving rates of cytoreduction. We report the results from the adoption of a multidisciplinary approach to EOC surgery in an attempt to overcome some of these barriers.

Our study describes surgical outcomes from consecutive, prospectively registered patients with EOC in a single region in Scotland, excluding selection bias. We report a combined complete and optimal $(<1 \mathrm{~cm})$ cytoreduction rate of $69.0 \%$. These data compare favourably with two large, randomized-controlled trials in women with advanced EOC where resection rates varied significantly between centres $[3,13]$ yet remain lower than those reported by other centres both nationally and internationally $[10,11,14]$. The median PFS of 14.3 months is similar to those reported in other UK populations and several international trials $[10,15]$. Despite a significant increase in the number of bowel resections $(20.5 \%$ in group 1 vs. $42.7 \%$ in group 2) and an associated reduction in pelvic residual disease, a more aggressive surgical approach did not significantly improve rates of complete or optimal cytoreduction and no significant improvement in progression free survival was seen between the two groups. This finding is in keeping with data from other groups which have suggested that incorporation of upper abdominal surgery is required if rates of cytoreduction are to be significantly improved. Chi et al reported a significant increase in rates of complete cytoreduction associated with a change in the surgical paradigm to include upper abdominal procedures in surgery for EOC, achieving optimal resection rates of $80 \%$ with an associated improvement 
in both PFS and OS as a result [14]. Similarly, in a UK setting, the two previously published reports on cytoreductive surgery demonstrated high rates of maximal cytoreduction following incorporation of upper abdominal procedures. Fotopoulou et al report complete cytoreduction in $89 \%$ of patients [10]. Turnbull at et al reported complete cytoreduction in $54.1 \%$ and optimal in a further $34.1 \%$ of women giving a combined rate of $88.2 \%$ [11]. Although a small number of upper abdominal procedures were performed in this series, complex, cytoreductive upper abdominal surgery was not regularly incorporated into surgical practice in this cohort in which twenty-five patients had single site residual disease in the upper abdomen. With the incorporation of surgical procedures in the upper abdomen in this cohort we suggest that rates of cytoreduction could have increased to $79.0 \%$.

Of note, while the operational changes introduced in our institution did not improve rates of complete or optimal cytoreduction, surgical outcomes were maintained while the number of patients undergoing primary surgery rather than interval debulking increased significantly. The EORTC 55971 and CHORUS trials both reported significantly lower rates of optimal cytoreduction in patients undergoing primary surgery $[3,14]$. In our population, a move towards more primary surgery did not result in a significant change in cytoreduction nor any increase in morbidity or mortality. Whilst these randomised trials demonstrated interval debulking to be non-inferior to primary surgery, the choice between these strategies remains controversial. A secondary analysis of the EORTC 55971 trial suggested that survival may be superior in selected patients undergoing primary surgery and data from retrospective studies have suggested that complete macroscopic resection at primary surgery may be associated with improved survival[16, 17]. This is currently being further examined in the TRUST trial, a randomized, controlled multicentre trial comparing radical upfront surgery to interval debulking surgery (clinicaltrials.gov, ID NCT02828618). In our study, primary surgery was associated with improved PFS compared to delayed surgery when examined across the entire study population. A significant improvement in PFS was also identified in group 1. No difference was identified in group 2, in which more patients underwent primary surgery, although follow up was noted to be significantly shorter in group 2 and thus this figure may subsequently change. Of course, the process of patient selection for primary surgery introduces some bias into this aspect of our analysis, however these results suggest that primary surgery should remain the preferred treatment option when possible.

A significant concern regarding the adoption of increasing radicality in EOC surgery relates to the potential for increased morbidity and mortality and the subsequent effect on quality of life. In our cohort, overall 30 -day and 60 -day mortality was low, $0.4 \%$ and $1.3 \%$, respectively. This compares to the $3.2 \% 28$ day mortality in similar settings in the CHORUS trial [13]. Similarly, our major morbidity rate of $10.3 \%$ was low and did not significantly change between the two groups (9.8\% vs 10.7\%) despite an increase in patients undergoing primary surgery. Centres with experience of more extensive surgery have reported major morbidity of around $20 \%[10,14,18]$. Quality of life data for this cohort has not been collected, the 
SOCQER-2 study, a prospective study examining quality of life following surgery for EOC is currently recruiting (clinicaltrials.gov, ID NCT02559983) examining the impact of radical cytoreductive surgery.

We acknowledge that the assessment of residual disease following surgery is difficult and there remains significant risk of underestimation of residual disease status. Chi et al found a $43 \%$ discordance between surgeon's assessment and postoperative CT assessment although this did not significantly affect median PFS [19]. By limiting this study to a single region some of the variability in the recording of residual disease status is reduced in comparison to studies investigating multiple sites.

In this study we attempted to introduce a collaborative system to overcome some of the barriers to maximal surgical effort identified in previous UK surveys. As a result, we have increased the radicality of surgery in the pelvis and mid abdomen and increased the number of patients undergoing primary surgery without significant increase in the associated surgical morbidity. Despite this, no significant improvement in residual disease status or consequently, PFS, was achieved (although the median follow up in group 2 of our study was somewhat short at 18 months). These findings support data from other authors indicating that upper abdominal surgery is required to achieve improvement in cytoreduction. These findings have enabled us to extend the surgical expertise in cases of advanced EOC to include support from colleagues in hepatobiliary surgery and we anticipate an associated improvement in cytoreduction as identified by other centres. While we recognise that alternative approaches have successfully been adopted in other centres we believe this approach represents a useful model for centres looking to co-ordinate institutional efforts to safely deliver maximal surgical effort in EOC.

Developing recognition of the cellular and molecular diversity of ovarian cancer may contribute to more refined selection of patients most likely to benefit most from such aggressive surgical management. In the meantime, the debate regarding primary surgery and neoadjuvant chemotherapy notwithstanding, if improvements in EOC are to be achieved in the UK, robust quality improvement strategies are required. Surgery with the aim of complete cytoreduction within healthcare models which support prolonged operating times and minimise morbidity must be established. When historic structural barriers such as institutional governance issues or surgical experience and training exist, collaborative multidisciplinary systems can be introduced with the aim of providing outcomes for UK patients equal to the best international results.

\section{Declarations}

\section{Ethics approval and consent to participate}

As a service evaluation this study is exempt from the requirement for research ethical review. Data collection, storage and analysis were in accordance with local governance protocols and authorised by the local Caldicott guardian.

\section{Consent for publication}


Not applicable

\section{Data statement}

The datasets generated and/or analysed during the current study will be available from the corresponding author on reasonable request.

\section{Conflict of interest}

The authors have nothing to declare

Funding

None

\section{Authors' contributions}

JM drafted the manuscript. All authors have read, critically reviewed for important intellectual content and approved the final manuscript.

\section{Acknowledgements}

The authors wish to acknowledge Mohamed Otify and Vanitha Sivalingam for their assistance in the collection of data.

\section{References}

1. Ovarian cancer statistics [http://cancerresearchuk.org/health-professional/cancer-statistics]. Accessed $26^{\text {th }}$ Januaray 2018

2. Chang SJ, Hodeib M, Chang J, Bristow RE. Survival impact of complete cytoreduction to no gross residual disease for advanced-stage ovarian cancer: a meta-analysis. Gynecologic oncology 2013, 130(3).

3. Vergote I, Trope CG, Amant F et al. Neoadjuvant chemotherapy or primary surgery in stage IIIC or IV ovarian cancer. The New England journal of medicine 2010, 363(10).

4. Sant M, Chirlaque Lopez MD, Agresti R et al. Survival of women with cancers of breast and genital organs in Europe 1999-2007: Results of the EUROCARE-5 study. European journal of cancer 2015, 51(15).

5. Coleman MP, Forman D, Bryant $\mathrm{H}$ et al. Cancer survival in Australia, Canada, Denmark, Norway, Sweden, and the UK, 1995-2007 (the International Cancer Benchmarking Partnership): an analysis of population-based cancer registry data. Lancet 2011, 377(9760).

6. Abdel-Rahman M, Stockton D, Rachet B, Hakulinen T, Coleman MP. What if cancer survival in Britain were the same as in Europe: how many deaths are avoidable? British journal of cancer 2009, 101 Suppl 2. 
7. Naik R, Bayne L, Founta C, Kehoe S, Rustin G, Fotopoulou C. Patient Support Groups Identifying Clinical Equipoise in UK Gynaecological Oncology Surgeons as the Basis for Trials in Ultraradical Surgery for Advanced Ovarian Cancer. International journal of gynecological cancer 2016, 26(1).

8. Barton DP, Adib T, Butler J. Surgical practice of UK gynaecological oncologists in the treatment of primary advanced epithelial ovarian cancer (PAEOC): a questionnaire survey. Gynecologic oncology 2013, 131(2).

9. Elattar A, Bryant A, Winter-Roach BA, Hatem M, Naik R. Optimal primary surgical treatment for advanced epithelial ovarian cancer. The Cochrane database of systematic reviews 2011(8):Cd007565.

10. Fotopoulou C, Jones BP, Savvatis $\mathrm{K}$ et al. Maximal effort cytoreductive surgery for disseminated ovarian cancer in a UK setting: challenges and possibilities. Archives of gynecology and obstetrics 2016, 294(3).

11. Turnbull HL, Akrivos N, Wemyss-Holden S et al. The impact of ultra-radical surgery in the management of patients with stage IIIC and IV epithelial ovarian, fallopian tube, and peritoneal cancer. Archives of gynecology and obstetrics 2017, 295(3).

12. Cibula D, Verheijen R, Lopes A, Dusek L. Current clinical practice in cytoreductive surgery for advanced ovarian cancer: a European survey. International journal of gynecological cancer 2011, 21(7).

13. Kehoe $\mathrm{S}$, Hook J, Nankivell $\mathrm{M}$ et al. Primary chemotherapy versus primary surgery for newly diagnosed advanced ovarian cancer (CHORUS): an open-label, randomised, controlled, non-inferiority trial. Lancet 2015, 386(9990).

14. Chi DS, Eisenhauer EL, Zivanovic $O$ et al. Improved progression-free and overall survival in advanced ovarian cancer as a result of a change in surgical paradigm. Gynecologic oncology 2009, 114(1).

15. Khoja L, Nolan K, Mekki R et al. Improved Survival from Ovarian Cancer in Patients Treated in Phase III Trial Active Cancer Centres in the UK. Clinical oncology 2016, 28(12).

16. van Meurs HS, Tajik P, Hof MH et al. Which patients benefit most from primary surgery or neoadjuvant chemotherapy in stage IIIC or IV ovarian cancer? An exploratory analysis of the European Organisation for Research and Treatment of Cancer 55971 randomised trial. European journal of cancer $2013,49(15)$.

17. Kessous R, Laskov I, Abitbol J et al. Clinical outcome of neoadjuvant chemotherapy for advanced ovarian cancer. Gynecologic oncology 2017, 144(3).

18. Aletti GD, Dowdy SC, Gostout BS et al. Quality improvement in the surgical approach to advanced ovarian cancer: the Mayo Clinic experience. Journal of the American College of Surgeons 2009, 208(4).

19. Chi DS, Barlin JN, Ramirez PT et al. Follow-up study of the correlation between postoperative computed tomographic scan and primary surgeon assessment in patients with advanced ovarian, tubal, or peritoneal carcinoma reported to have undergone primary surgical cytoreduction to residual disease of $1 \mathrm{~cm}$ or smaller. International journal of gynecological cancer 2010, 20(3). 


\section{Tables}

\begin{tabular}{lcccc}
\multicolumn{1}{c}{ Variable } & $\begin{array}{c}\text { Overall } \\
(\mathrm{n}=224)\end{array}$ & $\begin{array}{c}\text { Group 1 } \\
(\mathrm{n}=112)\end{array}$ & $\begin{array}{c}\text { Group 2 } \\
(\mathrm{n}=112)\end{array}$ & p value \\
\hline Median age (years) & $66(19-89)$ & $66(19-88)$ & $66(29-89)$ & NS \\
\hline FIGO stage at diagnosis & & & & \\
\hline III & $161(71.9 \%)$ & $81(72.3 \%)$ & $80(71.4 \%)$ & NS \\
IV & $63(28.2 \%)$ & $31(27.7 \%)$ & $32(28.5 \%)$ & \\
Histological subtype & & & & \\
\hline High grade serous & $177(79.0 \%)$ & $85(75.9 \%)$ & $92(78.6 \%)$ & NS \\
\hline Low grade serous & $13(5.7 \%)$ & $7(6.3 \%)$ & $6(5.1 \%)$ & \\
\hline Endometrioid & $6(2.6 \%)$ & $2(1.8 \%)$ & $4(3.4 \%)$ & \\
\hline Clear cell & $6(2.6 \%)$ & $3(2.7 \%)$ & $3(2.6 \%)$ & \\
\hline Mucinous & $1(0.4 \%)$ & 0 & $1(0.9 \%)$ & \\
\hline Carcinosarcoma & $14(6.1 \%)$ & $10(8.9 \%)$ & $4(3.4 \%)$ & \\
\hline Adenocarcinoma, unclassified & $2(0.9 \%)$ & $2(1.8 \%)$ & 0 & \\
\hline Mixed & $5(2.2 \%)$ & $3(2.7 \%)$ & $2(1.7 \%)$ & \\
\hline Primary treatment & & & & \\
\hline Surgery & $107(47.8 \%)$ & $44(39.3 \%)$ & $63(56.3 \%)$ & 0.02 \\
\hline Neo-adjuvant chemotherapy & $117(52.2 \%)$ & $68(60.7 \%)$ & $49(43.8 \%)$ & \\
\hline Median Follow up (months) & $23(1-60)$ & $37(2-60)$ & $18(1-39)$ & \\
\hline
\end{tabular}

Table 1. Patient Characterisitcs.

\section{Cytoreduction}

\begin{tabular}{c|ccc|ccc|cc}
\hline & \multicolumn{9}{c}{ Complete } & \multicolumn{2}{c}{$<1 \mathrm{~cm}$} & \multicolumn{2}{c}{ Complete } & $<1 \mathrm{~cm}$ \\
\hline & Group 1 & Group2 & $\begin{array}{c}\mathrm{p} \\
\text { value }\end{array}$ & Group 1 & Group2 & $\mathrm{p}$ & \multicolumn{2}{c}{ Combined } \\
& & & & & value & & \\
\hline Primary & $21 / 44$ & $24 / 63$ & $\mathrm{~ns}$ & $10 / 44$ & $14 / 63$ & $\mathrm{~ns}$ & $45 / 107$ & $24 / 107$ \\
Surgery & $(47.7 \%)$ & $(38.1 \%)$ & & $(22.7 \%)$ & $(22.3 \%)$ & & $(42.1 \%)$ & $(22.4 \%)$ \\
Interval & $32 / 68$ & $21 / 49$ & $\mathrm{~ns}$ & $15 / 68$ & $15 / 49$ & $\mathrm{~ns}$ & $53 / 117$ & $30 / 117$ \\
Debulking & $(47.1 \%)$ & $(42.9 \%)$ & & $(22.1 \%)$ & $(30.6 \%)$ & & $(45.3 \%)$ & $(25.6 \%)$ \\
Total & $53 / 112$ & $45 / 112$ & $\mathrm{~ns}$ & $25 / 112$ & $29 / 112$ & $\mathrm{n}$ & $98 / 224$ & $54 / 224$ \\
& $(47.3 \%)$ & $(40.2 \%)$ & & $(22.3 \%)$ & $(25.9 \%)$ & & $(43.8 \%)$ & $(24.1 \%)$ \\
\hline
\end{tabular}

Table 2. Rates of cytoreduction 


\section{Procedure}

Group 1 (\%) Group 2 (\%)

\begin{tabular}{lcc} 
& $(\mathrm{n}=112)$ & $(\mathrm{n}=112)$ \\
\hline Large bowel resection & $20(17.89)$ & $40(35.7)$ \\
\hline Small bowel resection & $4(3.6)$ & $6(5.4)$ \\
\hline Ileostomy & $5(4.5)$ & $9(8.0)$ \\
\hline Colostomy & $6(5.4)$ & $14(12.5)$ \\
\hline Lymph node dissection (inguinal/pelvic/para-aortic) & $9(8.0)$ & $14(12.5)$ \\
\hline Parietal peritonectomy & $8(7.1)$ & $29(25.9)$ \\
\hline Abdominal wall resection & $1(0.9)$ & $4(3.6)$ \\
\hline Splenectomy & 0 & $5(4.5)$ \\
\hline Cholecystectomy & $1(0.9)$ & $1(0.9)$ \\
\hline Partial gastric resection & 0 & $1(0.9)$ \\
\hline
\end{tabular}

Table 3. Surgical procedures

\begin{tabular}{lcccc} 
Morbidity & & Group 1 & Group 2 & Overall \\
\hline Return to theatre & $\mathrm{n}=112$ & $\mathrm{n}=112$ & $\mathrm{n}=224$ \\
\hline & Haemorrhage & 1 & & 1 \\
\hline Post operative readmission & Small bowel obstruction & & 1 & 1 \\
\hline Anastomotic leak & 1 & 2 & 3 \\
\hline Wound infection/Dehiscence & 2 & 1 & 3 \\
\hline Lower Respiratory Tract Infection & 1 & 2 & 3 \\
\hline Urosepsis & 6 & 4 & 10 \\
\hline CVA & & 1 & 1 \\
\hline Total & $11(9.8 \%)$ & $12(10.7 \%)$ & $23(10.3 \%)$
\end{tabular}

Table 4. Morbidity

Figures 


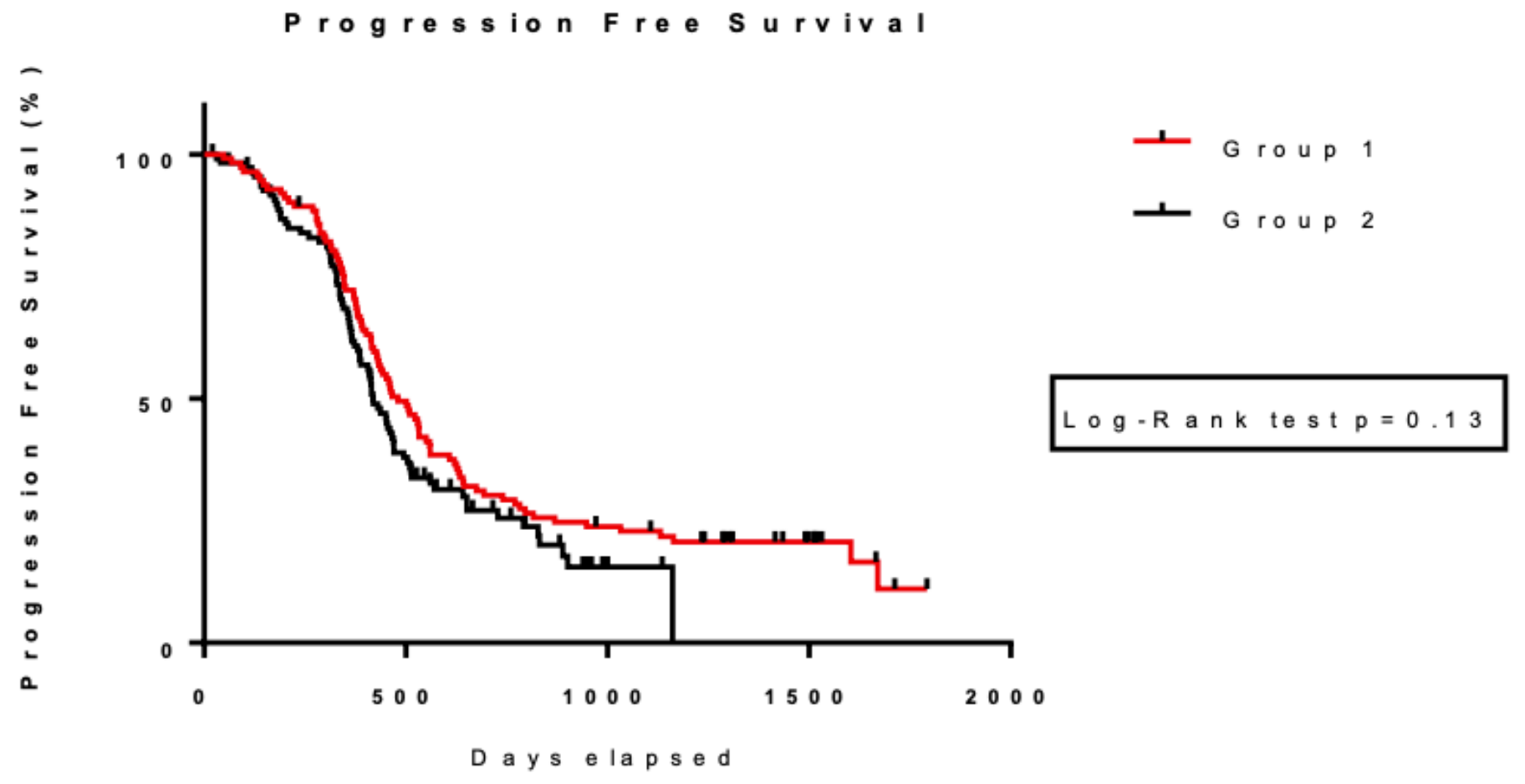

Figure 1

Progression Free Survival

Prim a ry s u rgery v s N A C T

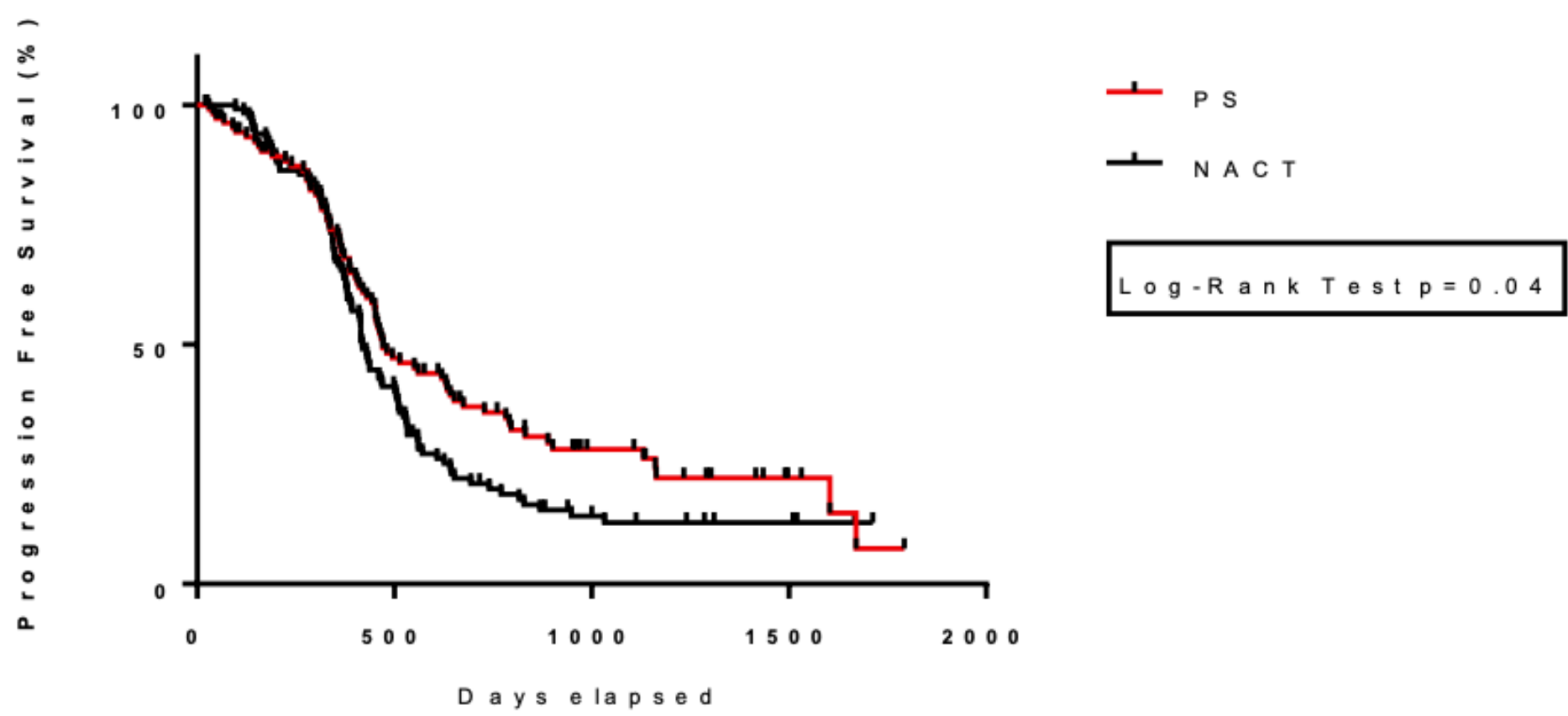

Figure 2

Primary surgery versus Delayed surgery (whole study population) - Progression Free Survival 
G $r \circ u$ p 1

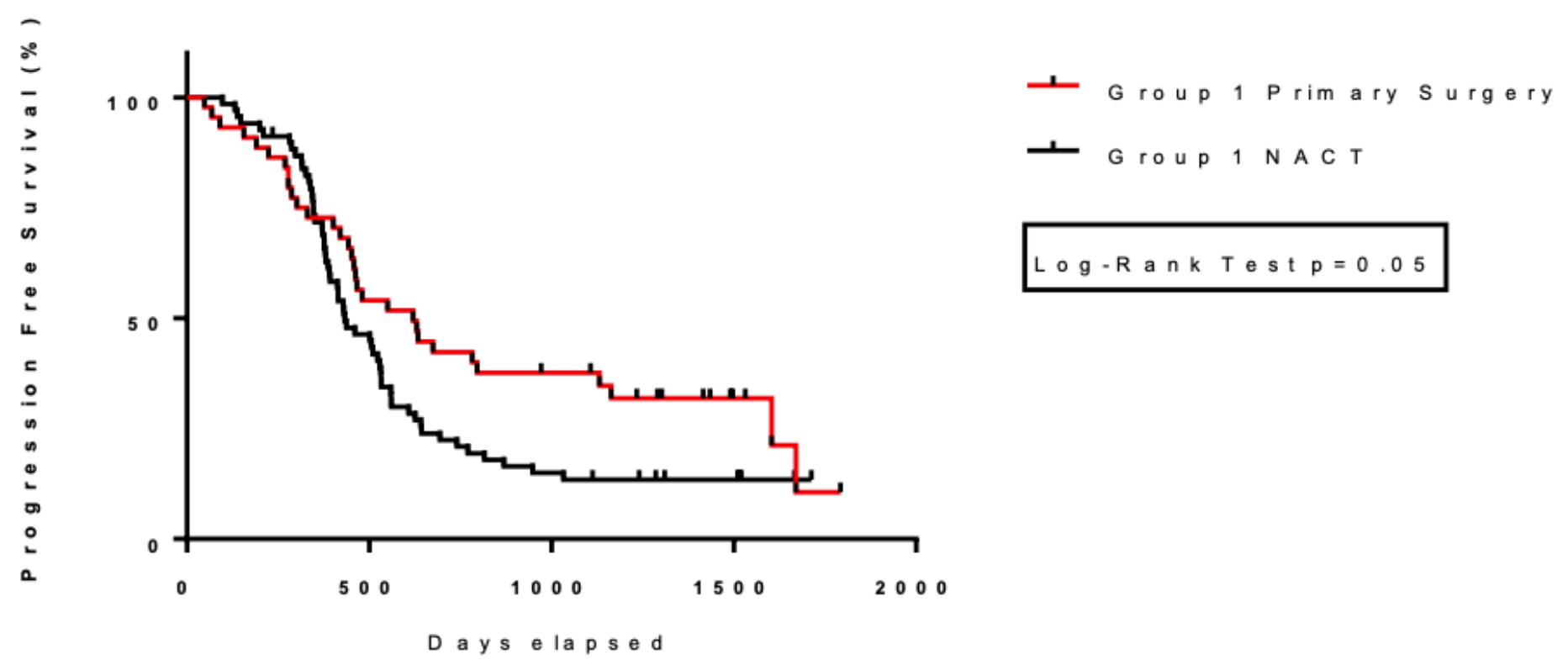

Figure 3

Primary surgery versus Delayed surgery (Group 1) - Progression Free Survival

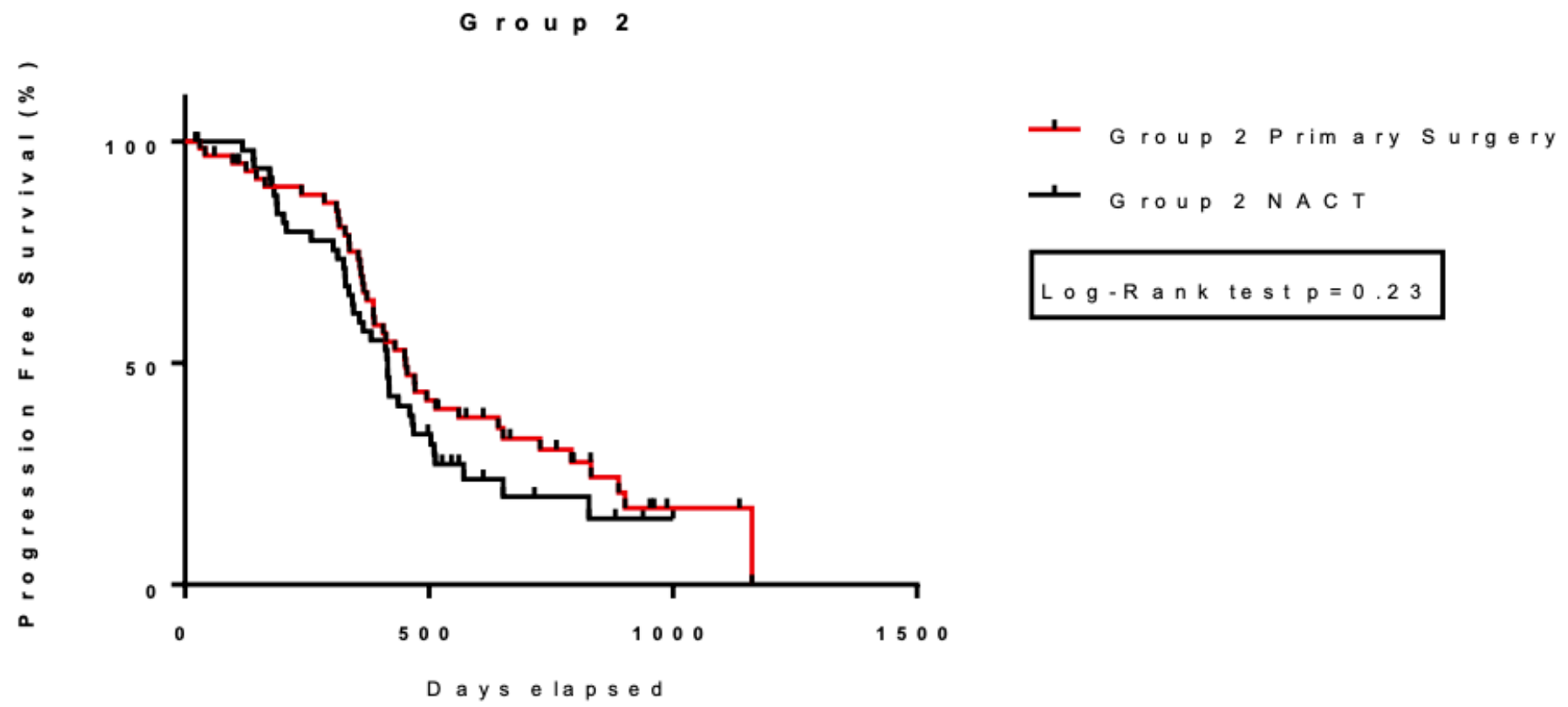

Figure 4

Primary surgery versus Delayed surgery (group 2) - Progression Free Survival 